\title{
SPEECH EXPERIENCE IN A STRUCTURE OF THE SOCIAL INTELLIGENCE
}

\section{Orap Maryna ${ }^{1}$}

DOI: http://dx.doi.org/10.30525/978-9934-571-27-5_35

\begin{abstract}
The study deals with the psychological aspects of problem of interconnection between social intelligence and speech experience. The analysis of the general theoretical and methodological principles of research the social intelligence and speech experience are given. Research main goal is in definition the methodological prepositions in studying social intelligence in its connection with personality's speech experience. In the article solves the following tasks: 1) clarification the methodological differences in the study of social intelligence and definition the conceptual and essential provisions for constructing the theoretical and empirical study the regularities of speech prepositions of social intelligence; 2) analysation the results of pilot empirical research in order to find correlations between the level of development of speech experience and the level of prognostic possibilities in the structure of social intelligence. It is determined that the internal structure of speech experience serves for fastening, regulation and systematizing of speech mastery of the world results by means of speech capability, speech abilities, speech competence and speech activity. It is cleared up that the elements of the internal structure are connected with one another in horizontal and vertical planes.

Empirical study predicted two directions: 1) studying the level of speech experience; 2) studying the social intelligence level of each respondent. Statistically significant correlations between indicators of speech experience and social intelligence give the opportunity to make a decision about their interconnection. Elements of the internal structure of the speech experience show mutual relationship with prognostic abilities in social interaction.
\end{abstract}

In personal aspect of speech experience functioning, quantitative level of speech experience organization are replaced with indicators of individ-

${ }^{1}$ Doctor of Psychology, Associate Professor,

Professor of Department of Psychology,

Volodymyr Hnatiuk Ternopil National Pedagogical University, Ukraine 
ual style of combination of personal traits with structural and functional peculiarities of speech experience. So, individual peculiarities of speech experience lead to individual characteristics of personality's social connection. It was proved that the features of the social intelligence were largely determined by personal level of speech ability, speech capabilities, speech competence, speech activity and by the level of its organization into system. The analysis of the correlation relations between the levels of organization of the elements of the internal structure of the speech experience and social intelligence capabilities demonstrates the existence of statistically significant. This allows us to assert the mutual influence of speech experience and social intelligence.

\section{Introduction}

The problem of social intelligence has been intensified in scientific psychological theoretical and practical researches in connection with the latest requirements for the personality's social life. At the forefront of today's personality are the requirements of flexibility in social relations and the speed of adaptation to new conditions. The analysis of modern Ukrainian and foreign researches had shown an increase interest to the problem of social intelligence, in its various ontogenetic, structural and functional features. At the same time, it made it possible to distinguish an interesting pattern. The question of the content, functions and structural elements of social intelligence (as, and in general, intellect) remains actual in scientific literature. And therefore, he has a dozen different studies and accentuations on certain aspects of the issue under consideration. In this case, all researchers in the empirical study use the same diagnostic tool (mainly, the test of J.P. Guilford), forgetting that it was created on a specific methodological basis from the theory of social intelligence, which differs from other theories. That is why we are seeing a situation where the authors, using social intelligence as an ability to adapt or socialize, use the battery of J.P. Guilford's tests based on the theoretical understanding of social intelligence, in the first place, as knowledge of behavior. Thus, we were seeing a methodological mistake, which, in our opinion, is the reason of the lack of social intelligence fundamental researches. We believe that the avoidance of this mistake is possible by a clear definition the methodological foundations of the research of social intelligence. 


\section{The analysis of modern researches}

Ukrainian researches characterized mainly by studying the applied aspects of social intelligence, such as: the patterns and peculiarities of formation the social intelligence children of the junior school age (A.A. Melnik) and adolescents (M. Falchuk), specialists of socio-occupational professions (Y.Y. Kaplunenko), bilingual students (N.O. Burayiko), future psychologists (L.O. Lyakhovets), managers (G.V. Ozhbko) and heads of educational institutions (S.I. Redko). Social intellect is considered as a precondition for successful pedagogical activity (S.V. Rudenko, E. Ivashkevich). It should be noted that modern Ukrainian comprehensive research of social intelligence, carried out by E.Z. Ivashkevich, embodied in the monograph "Social Intelligence of the Teacher" [1]. There is also an active attention to close problems: social abilities (O.I. Vlasova), social potential and social competence (N.V. Kazarinova, V.M. Pogolsha), adaptation (G.S. Altunin, N.I. Bulka, N.L. Kaminska) and perception (V.M. Kunitsina), the effectiveness of social interaction (A.K. Mudrik), which are considered as components of social intelligence. Thus, adaptive, communicative, and creative components are emphasizing in social intelligence as responsible for the effectiveness of social interaction.

The very close correlation between the concept of social intelligence and socio-psychological categories such as socialization, social adaptation, social competence and social skills leads to difficulties in definition of social intelligence. Different studies of social intelligence (SI) include and underline aspects such as: social knowledge (N. Cantor, J. Kilstrom, S. Wong, J. Day, S. Maxwell, N. Mir, S. Kosmith, C. John); social memory (J. Washington); social perception (R. Riggio) and anticipation (J.P. Guilford, S. Kosmitsky, O. John); social or communicative competence (M. Ford, M. Tisac, N. Kentor); social interpretation as deciphering non-verbal information (R. Anthony, R. Rosenthal, D. Archer, R. Arket, R. Sternberg, J. Smith); social intuition (F. Chapin, S. Wong, J. Dey, D. Keating, R. Rosenthal); social understanding (Y.M. Emelyanov); social adaptation (G.W. Allport, D. Kitting, F. Chapin, N. Kentor, R. Harlow); social and cognitive flexibility (C. John, J. Day). The variety of socio-psychological phenomena that are involved in the problem of social intelligence, and, sometimes completely replace its content, lead to the thoughts about the absence of this kind of intelligence as an independent psychological phenomenon. This, of course, complicates the process of his scientific study. 
Thus, the analysis of recent publications shows two tendencies, which determine the relevance of this problem and the purpose of the study. On the one hand, modern society requires researches which explain the precondition of successful social interaction. On the other hand, we do not see enough research about the role of speech activity in development and functioning of social intelligence.

\section{Research purpose}

Research purpose is in definition the methodological prepositions in studying social intelligence in its connection with personality's speech experience. So, this article solves the following tasks: 1) clarification the methodological differences in the study of social intelligence and definition the conceptual and essential provisions for constructing the theoretical and empirical study the regularities of speech prepositions of social intelligence; 2) analysation the results of pilot empirical research in order to find correlations between the level of development of speech experience and the level of prognostic possibilities in the structure of social intelligence.

\section{Methods and techniques}

In research were used analytical-synthetic and comparative theoretical methods; J.P. Guilford's method "Diagnostics of social intelligence" [2]; author's methodology for studying the level of speech experience organization [3].

\section{Basic statements. Theoretical preposition}

The term "social intelligence" was introduced into the psychology of E. Torndike in 1920 to refer to "foresight in interpersonal relations" in one row with abstract and specific intelligence. "Social intelligence shows itself abundantly in the nursery, on the playground, in barracks and factories and salesrooms, but it eludes the formal standardized conditions of the testing laboratory" - wrote Edward Thorndike in a 1920 article in Harper's Monthly Magazine. E. Thorndike noted that such interpersonal effectiveness was of vital importance for success in many fields, particularly leadership. "The best mechanic in a factory," he wrote, "may fail as a foreman for lack of social intelligence."

But, as J.P. Guilford said, "Thorndike's "social intelligence" and Cross's "work with - people" are too general to be of practical value over and above 
naming the domain of interest" $[9$, p. 12]. Observing people interacting with other people suggests that there are a number of different ways of being socially intelligent. As M. Argyle has noted, "clearly most people are better at some social tasks than others... There are, for example, people who are better at handling audiences, or committees, than at dealing with individuals - or vice versa" [5, p. 77]. Some people are astute in understanding or cognizing what others think and feel, but for reasons of timidity or poor social training do not behave well in social situations. Others, while not particularly perceptive of others' feelings and thoughts, are socially poised and well-informed. Some individuals, such as successful statesmen, can produce many different solutions to a social problem.

Due to the researches by J.P. Guilford, the term "social intelligence" has become a category of measured constructs, was entered into an arsenal of psychological practice. The basis for this was the theoretical considerations of J.P. Guilford, according to which social intelligence is a system of intellectual abilities that are relatively independent of the factor of general intelligence and are strongly related to the knowledge of behavioral information. The structure of intellect postulates 120 different factors of intellectual ability organized along three dimensions: content (semantic, symbolic, figural and behavioral); operation (cognition, memory recoding, memory retention, divergent production, convergent production and evaluation); and product (units, classes, relations, systems, transformations and implications). Within the behavioral content area, Guilford hypothesizes 30 different intellectual abilities categorized according to the five intellectual operations and six products involved. For example, persons with the ability to think of many different social solutions or to generate many different facial expressions might be described as gifted in the area of behavioral divergent production. Those who make wise social decisions are good behavioral evaluators, while those who never forget an interpersonally relevant occurrence have superior behavioral memory. This study demonstrates the existence of six factors of social intelligence dealing with the ability to understand other people's thoughts, feelings, and intentions. These are called factors of "behavioral cognition" to indicate that they refer only to the abilities to cognize or understand behavior, and not to other abilities which might more broadly and inclusively be termed "social intelligence." Therefore, behavioral cognition is more fully defined as the ability to understand the thoughts, feelings and intentions of other people insofar as they 
are manifested in discernible behavior. Although behavioral cognition is probably most adequately demonstrated in real-life situations, economy dictated tests which used less than life-size stimuli. Further studies either complemented the theory of J.P. Guilford, or attempted to determine the independent predicates of social intelligence.

\section{Social intelligence: discussion theoretical questions}

The analysis of theoretical literature highlighting the main discussion issues in the SI problem, which demonstrate and determine the differences in methodological approaches to its study.

1. The first is the question of the designation the concept and definition the structure of the psychological phenomenon. The problem also lies in the fact that social intelligence can be showing up both in personality features and in intellectual peculiarities. This, in our opinion, leads to a variety of approaches to the definition of the nature of the SI. As a result of the analysis of theoretical literature, the following approaches can be distinguished: a) SI as an understanding of oneself and other people, based on the specifics of mental processes, affective responsiveness and social experience (Y. Yemelianov); b) the ability to distinguish the essential characteristics of the communicative situation that are not directly observable (J.P. Guilford, L.S. Vygotsky, D.B. Yelkonin, O. Chesnokova); c) SI as ability - as a component of cognitive (N. Kalina), social-perceptual (V. Labunskaya, A. Yuzhaninova), social (O. Vlasova), which is a part of "social talent" of personality (G.W. Allport, O. Bodalov) as a global ability - communicative-personal potential (V. Kunitsina); as a special "social gift" (G.W. Allport); d) SI as a set or system of social knowledge (N. Kentor, J. Kilstrom, S. Wong, J. Dey, S. Maxwell, N. Mir, S. Kosmitsky, C. John, D. Ushakov), which provide ( is a prerequisite) a high level of social competence (M. Ford, M. Tisac); e) SI as skills or abilities of social adaptation (G.W. Allpor, D. Kitting, F. Chapin, R. Harlow, etc.) or social interpersonal perception (G.M. Andreyev); e) SI as a structure of personal experience that allows solving problems of social life (N. Kentor).

2. The second question, which is the key to choosing a research direction, is the question of interconnection and interaction of general (academic, abstract, practical) intelligence and social intelligence. As E.Z. Ivashkevich notes, in addition to the question of the essence of social intelligence, the problem of correlation of it with the general intellect, intellectual talent, 
etc. [1, p. 21]. In this question, based on the analysis of literature, we have identified three main approaches. First, social intelligence is seen as a kind of general intelligence. The main direction of such studies is through the comparison of general and social intelligence. Diagnostics of SI in the whole repeats traditional tests of intelligence, but characterized by much cognitive-verbal orientation. R. Rijgio, D. Kitting, J. Vedek worked in this direction, but the work with verbal material did not make it possible to differentiate their own essential predictors of SI. Especially their experiments have revealed the independence between the verbal part of the general intelligence and social intelligence.

In the interpretation of H.J. Eysenck social intelligence - is the intelligence that "grows" from the general intelligence, formed during the socialization of the individual. Therefore, it determines the general features of cognitive (including social) human activities formed in the process of its socialization. As R.E. Riggio said, "intelligence or IQ, is largely what you are born with. Genetics play a large part. Social intelligence (SI), on the other hand, is mostly learned. SI develops from experience with people and learning from success and failures in social settings. It is more commonly referred to as "tact," "common sense," or "street smarts" [11].

The second direction considers the SI as an independent form of intelligence, which ensures the adaptation of man in society and directional at solving life problems. Both behavioral and non-verbal components of solving social problems are used. Studies by J.P. Guilford and Maureen O'Sullivan, M. Ford, L. Braun, R. Selman have shown that the level of SI differs from the academic one, but there is a potential correlation between them. Further in studies by J. Wedek (Wedeck, J. 1947), it was proved that SI does not significantly correlate with the development of general intelligence and spatial representations. It should be emphasized separately that in the J.P. Guilford's studies social intelligence did not significantly correlate with the level of development of the general intelligence and spatial ideas, the ability to visualize the distinction and the originality of thinking.

In the third approach SI is represented as an integral ability to communicate based on personal characteristics and levels of self-awareness. The emphasis is on the communicative orientation of the SI, and thus aimed at measuring personal characteristics that correlate with the parameters of social maturity (N. Kentor, M. Bobnev, V. Kunitsina). 
As a result of the analysis of the content contributed by different authors in the definition of SI, we came to the conclusion that the specific one that describes the reality of the SI itself (but not social competence, social adaptability, social interaction skills, etc.) is precisely prediction - prediction the development of the scenario and the results of social events. In the current research carried out in Russia, in particular under the guidance of D.V. Ushakov and T.G. Leontiev, correlations of indicators of social intelligence and personality characteristics of children of junior school age were revealed. It was concluded that the high level of development of the SI is combined with the presence of such traits as: self-confidence, propensity to self-affirmation, aspiration for leadership, ease of communication. Thus, was detected a tendency that raising the level of social intelligence contributes to the growth of confidence, independence, determination and ease of communication. It was also found that benevolence, intelligence, balance, carelessness, kindness and social normality are directly and closely not related to social intelligence.

But if we turn to the definition of intelligence as "the cognitive ability to all levels of reflection, prediction and rational transformation of reality, that permeates all spheres of human life and activities of the subject of knowledge" [4, p. 9], then the most appropriate is the consideration of the SI as a cognitive ability to reflect, predict and appropriate transformation of circumstances, subjects and content of social interaction. Thus, we define social intelligence as an ability that arises on the basis of a complex of intellectual, personal, communicative and behavioral features and predict the development of interpersonal situations, the interpretation of information and behavior, readiness for social interaction and decision-making. All phenomena analyzed above (social knowledge, skills, abilities, competence, ability, etc.) in this case, are considered predictors of the successful functioning of the SI. In this aspect, social intelligence involves the free possession of verbal and non-verbal means of social behavior, which places the role of speech experience in the development of the SI on the forefront.

Modern researches show that social intelligence is closely related to cognition and emotional intelligence $[6,7,8,12]$. "Psychologists argue about which human abilities are social and which are emotional" [6, p. 85]. Daniel Goleman in his book "Emotional Intelligence" folded social intelligence into the model of emotional intelligence. As he said, "I've come to see, simply lumping social intelligence within the emotional sort stunts fresh 
thinking about the human aptitude for relationship, ignoring what transpires as we interact" [8, p. 23].

According to D. Goleman the ingredients of social intelligence can be organized into two broad categories: social awareness (what we sense about others) and social facility (what we then do with that awareness). Social awareness refers to a spectrum that runs from primal empathy (instantaneously sensing another's inner state) to empathic accuracy (understanding her feelings and thoughts) to social cognition ("getting" complicated social situations). But simply sensing how another feels, or knowing what they think or intend, does not guarantee fruitful interactions. Social facility builds on social awareness to allow smooth, effective interactions. The spectrum of social facility includes self-presentation, influence, concern, and synchrony (interacting smoothly at the nonverbal level). Both the social awareness and social facility domains range from basic, "low-road" capacities, to more complex "high-road" articulations. Later K.A. Crowne added to the field of social and emotional intelligence also cultural intelligence and created the integrated model of intelligence that impact future research and managerial use of these constructs [6].

\section{Social intelligence and speech experience: \\ points of intersection and interconnection}

So, the analysis of social intelligence researches put the question about the role of speech, speech activity or language skills in development and functioning of social intelligence. What part of social communication skills is depending on personality's speech experience? Can person with low level of speech be successful in social intelligence abilities? As R. Riggio said, the key elements of social intelligence are: 1) verbal fluency and conversational skills; 2) knowledge of social roles, rules and scripts; 3 ) effective listening skills; 4) understanding what makes other people tick; 5) role playing and social self-efficiency; 6) impression management skills [11, p. 651]. So, as we can see, social intelligence is based on speech skills, such as verbal fluency, listening and conversational abilities.

Speech and language are vital for the development of emotional and social skills. Communication is one of the most important developmental tasks, which takes place during early childhood development. It is then; children begin to form their initial understanding of the world. Modern studies research the question about role of the speech in social interaction 
in different ways. Very important research was made by Sik H. $\mathrm{Ng}$ and James J. Bradac in their book "Power in Language: Verbal Communication and Social Influence" (1993). They seek to reveal how and why "the seemingly casual, routine use of language can recreate, enact, or otherwise subvert influence and control" [10, p. 3]. In approaching this task the authors explore and summarize a wide range of empirical research, discuss methodological issues, and consider power and influence in communicative contexts ranging from monological speech to conversational interaction to institutional discourse. So, we have a comprehensive analysis of research and theory on verbal communication and social influence. It examines a variety of empirical studies, theoretical positions, methodological matters and substantive issues pertaining to the use of language for generating influence and control. It moves from the basic concept of monological speech and the achievement of power to the increasingly complex and subtle cases of conversational control and linguistic depoliticization.

This study dedicated mostly to the problem of language behavior in social interaction and don't raises questions of the role of speech in social intelligence. On the other hand, as we can say, psychological researches in psycholinguistic field devoted mostly the problems of speech disorders of children and its influence on social development and the role of speech experience in studying foreign language.

Our recent study based on the theory of speech experience. Analysis of theoretical literature and practice of speech development leads us to believe that all the above phenomena that describe the complex mechanism of human speech, are the units of a higher order. Thus, we come to the idea of speech experience, which we see as individual speech system, which enables the speech mastering of the world [3]. It was making the structural and functional model of speech experience system with separation of internal and external structures and their elements. It is determined that the internal structure of speech experience serves for fastening, regulation and systematizing of speech mastery of the world results by means of speech capability, speech abilities, speech competence and speech activity. The elements of the internal structure are connected with one another in horizontal and vertical planes. The external structure of speech experience functions as the linguistic worldview, speech culture and speech competence interaction, which realize general function of external structure through the interpretation, interaction and integration 
functions - mediation of personality's speech mastering of the world. The regularities of internal and external structures and language experience interactions are proved by empirical data.

The main characteristic of the speech experience is defined by its organization, which is studied both as a process and as a property of the system. In the procedural aspects the speech experience is in the interaction of integration and differentiation processes. The properties of speech experience dynamics consists in increase of the height of the organization and the degree of organization, which leads to the system regulation and improvement.

The level of speech experience reveals correlation of emotional and strong-willed, communicative and intellectual traits of personality. This research posed new questions about the role of speech experience in person's social interaction and social intelligence in a whole. Can person have high level of SI without enough level of development speech experience?

We want to underline that we make strong difference between speech experience and social communication skills. Of course, they are related and influence each other. Communicative skills are considered as the basis of social interaction. Based on the experience of speech mastering of the world, communicative skills contain, also non-verbal components such as body language, gestures and interpersonal space. And in this aspect, communicative skills are more widely studied in their interrelation with social intelligence (K. Albrecht, J. Bell, M. Bobneva, E. Ivachkevych, C. MacLeod, E. Orlova, O. Smirnova, J. Tuhovsky). We emphasize the role of verbal components, which are united in the personality's speech experience.

\section{The empirical study}

In order to test the theoretical considerations, we carried out an experimental empirical study aimed at revealing the interrelationships between the level of development of social intelligence and speech experience. The sample was compiled by students of the Ternopil National Pedagogical University $(\mathrm{n}=116)$, the average age is 19 years.

Empirical study predicted two directions: 1) studying the level of speech experience; 2) studying the social intelligence level of each respondent.

1) Studying of speech experience we made on the basis of idea of organization and functioning the personality's speech experience. Speech expe- 
rience is a system in the psyche of the individual, which is the process of processing and ordering the results of speech mastering of the world. Personality's language view of the world, speech competency and speech culture are the products of such mastering. Analysis of speech development from the standpoint of child's speech experience allows describing its structure as a system of interconnected functional elements. This allows splitting the process of speech development for constituents that determine its dynamic and analyze each of them separately.

We based on the idea of L.S. Vygotsky that units of the structure are components, each of which reflects the characteristics of the whole structure. So, we define units of the structure of the speech experience: 1) speech ability, 2) speech capabilities, 3) speech competence and 4) speech activity. Each of this unit shows the level of development the speech experience as a whole.

Physiological precondition of the speech experience is speech ability. Speech capabilities cause the individual expression of personality's speech ability and formation of speech competence and activity. Learning of language skills and knowledge of the language creates personality's individual set of language and speech knowledge that combined in speech competence. This language means and manner of speech are forming and formulated thought that is the central link of the speech activity.

Thus, the structure of the speech experience presented by the speech ability, speech capabilities, speech competence and speech activity. In this aspect speech ability determines the potential possibility of speech activity; speech capabilities determine the individuality in mastering the knowledge of speech (speech competence) and formation of speech skills (speech activity).

I. Speech ability.

We studied speech ability through the diagnostic indicators and contained six techniques that were diagnosed: 1) language and speech disorders, 2) understanding relationships between words, 3) understanding of verbal material, 4) logical characteristics of associative reactions, 5) perception and understanding of speech, 6) willingness to produce speech. Determining the level of each indicator speech given ability to determine the general level of speech ability as an overall results indicator. So, a high level of speech ability was diagnosed in $24.74 \%$ of respondents, average $53.61 \%$, the lowest - in $21.65 \%$ of respondents. 


\section{Speech competence.}

Empirical referents of speech competence were defined: 1) the level of operational knowledge; 2) understanding language patterns (implicit knowledge); 3) perception and understanding of speech patterns; 4) the level of language proficiency and 5) correct vocabulary (declarative explicit knowledge); 6) knowledge of rules of the use of language units and 7) arbitrary speech (procedural knowledge). The general level of speech competence was determined by levels: medium group rate $(M)=13.021$, high levels defined within: $16-20$ points; the average $-11-15$ points, low -10.6 points. This allowed determining the level of speech competence.

III. Speech capabilities.

Speech capabilities were studied through diagnosis: 1) the level of verbal thinking, 2) originality of the images, 3) semantic flexibility 4) plasticity of speech, 5) verbal creativity 6) divergent productivity on verbal material, 7) initiative in speech activity. Transfer the diagnostic techniques results in points gives us the general level of speech capabilities: medium group rate $\mathrm{M}=10.360$; high level: $13-18$ points; the average, 9.12 points; low: 3-8 points. Analysis of the general level of skills of speech shows that the largest percentage of respondents characterized by average $-52.58 \%$ level. High levels observed in $22.68 \%$ and the lowest - in $24.74 \%$ of the respondents.

IV. Speech activity.

The level of speech activity was studied by diagnosis 1) the number of motives communication, 2) analysis of the precision of speech skills, 3) the rate of writing, 4) the level of speech facilities, 5) the level of allocation of essential features of meanings, 6) level of integration of meanings (characteristic grammatical associative reactions), 7) the level of understanding of metaphorical words, 8) features a frame, 9) features the script. The general level of speech activity is determined by means of conversion the marks into balls and levels: medium group rate $(M)=17.113$, high: $20-26$ points; the average: $15-19$ points; low: $8-14$ points. Accordingly, revealed that $24.74 \%$ of children characterized by a high level of speech activity, 53.61\% - average and 21.65 - a low level of speech activity.

An empirical study of social intelligence was carried out using subtests № 3 and № 4 by J. Guilford [2]. Guildford's technique contains four subtests that diagnose four abilities in the structure of SI: knowledge of classes, 
system knowledge, transformations and behavioral outcomes. Two subtests in their factor structure also have two additional meanings that relate to the ability to understand elements and attitudes. Subtest № 1 - Stories with completion - identification of feelings, emotions, intentions of participants in the situation, subtest № 2 - Expression groups - definition of mental states by the meaning of poses, gestures, facial expressions; Subtest № 3 - Verbal expression - prediction of an adequate verbal response in the communication situation; Subtest № 4 - Stories with additions - prediction of the most adequate scenario of the development of events in the social situation.

In subtest № 3, respondents need to choose from the proposed communication options the case in which this phrase will be different from the original meaning. Thus, the ability to understand the meaning of verbal reactions in different communicative situations is manifested. People with high ratings on this subtest are characterized by high sensitivity to the nuances of interpersonal relationships, helping them quickly and correctly understand what people are talking to each other. This subtest implies the existence of the speech experience as a necessary condition for its implementation.

Subtest № 4 - "Stories with additions” - built on non-verbal material measures the ability to understand the logic of the development of interaction situations, the importance of human behavior in these situations. People with high estimates for this subtest, able to recognize the structure of interpersonal situations in the dynamics, analyze the difficult situations of human interaction.

An analysis of the results of these subtests made it possible to divide the respondents into groups, depending on the level of development of social abilities. Quantitative ratios are shown in Diagram 1.

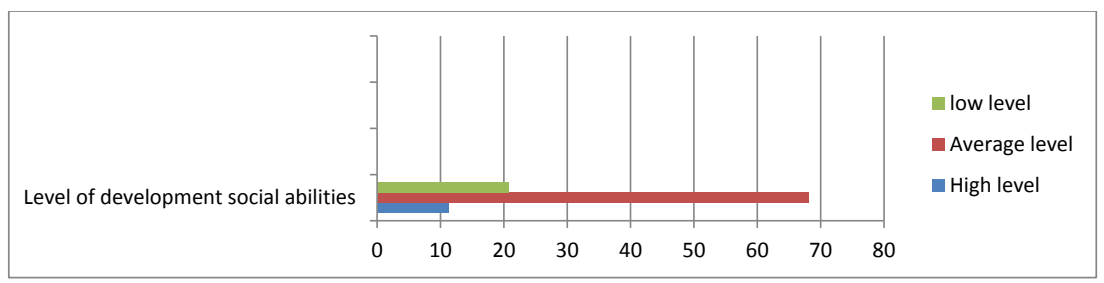

Diagram 1. The ratio of respondents to the level of development of social abilities 
Correlation analysis allowed us to say that the level of development of speech is interconnected with the development of social intelligence. There were statistically significant positive correlations between the indicators of the structural elements the internal structure of the speech experience (speech ability, speech capabilities, speech competence, speech activity) and verbal submissions "Verbal Expression" and "Stories with Addition", as shown in Table 1.

Table 1

\section{Correlation between the indicators of the general level of organization the internal structure of speech experience and the level of development of social intelligence}

\begin{tabular}{|l|c|c|}
\hline & $\begin{array}{c}\text { Prediction of an adequate } \\
\text { verbal response in the } \\
\text { communication situation }\end{array}$ & $\begin{array}{c}\text { Prediction of the most adequate } \\
\text { scenario of the development of } \\
\text { events in the social situation }\end{array}$ \\
\hline Speech ability & $0,401^{* *}$ & $0,398^{* *}$ \\
\hline Speech capabilities & $0,428^{* * *}$ & $0,379^{* *}$ \\
\hline Speech competence & $0,521^{* * *}$ & $0,498^{* * *}$ \\
\hline Speech activity & $0,563^{* * *}$ & $0,531^{* * *}$ \\
\hline
\end{tabular}

Note: ${ }^{*}-\mathrm{p}<0,05 ;{ }^{* *}-\mathrm{p}<0,01 ;{ }^{* * *}-\mathrm{p}<0,001$.

The analysis of the correlation relations between the levels of organization of the elements of the internal structure of the speech experience demonstrates the existence of statistically significant (levels of significance $\mathrm{p}<0,01$ and $\mathrm{p}<0,001)$ relationships between all the identified elements. This means that there are interconnections and interdependencies between all elements of the structure: changes to one element cause changes to other elements.

The logic of empirical research led to the make differentiation of respondents into groups according to the level of organization (high, average and low level) of the speech experience and compares them by the level of development of their social abilities. Significant correlations between the general level of organization of speech experience and the level of development of indicators of social intelligence have been shown in Table 2.

The qualitative analysis of empirical data provided important conclusions. The high level of organization of the speech experience does not always determine the success of predicting the most adequate scenario of 
Table 2

Correlation between the indicators of the general level of development of speech experience and the level of development of social intelligence

\begin{tabular}{|l|c|c|}
\hline & $\begin{array}{c}\text { Prediction of an adequate } \\
\text { verbal response in the } \\
\text { communication situation }\end{array}$ & $\begin{array}{c}\text { Prediction of the most } \\
\text { adequate scenario of the } \\
\text { development of events } \\
\text { in the social situation }\end{array}$ \\
\hline $\begin{array}{l}\text { High level of speech } \\
\text { experience }\end{array}$ & $0,433^{* * *}$ & $0,401^{* *}$ \\
\hline $\begin{array}{l}\text { Average level of speech } \\
\text { experience }\end{array}$ & $0,481^{* * *}$ & $0,434^{* * *}$ \\
\hline $\begin{array}{l}\text { Low level of speech } \\
\text { experience }\end{array}$ & $0,391^{* *}$ & $0,355^{* *}$ \\
\hline
\end{tabular}

Note: ${ }^{*}-\mathrm{p}<0,05 ; * *-\mathrm{p}<0,01 ; * * *-\mathrm{p}<0,001$.

the development of events in the social situation. A group of respondents with an average level of speech experience found a higher correlation with the ability to determine the sequence of situation logic. We explain this with a certain impulsiveness of the respondents with "fast" speech. The low level of development of speech experience has small correlation index with the level of prognostic ability. This means that the lack of development of speech does not prevent the development of social intelligence, but the high level of development of social intelligence determines the high level of development of speech experience.

\section{Conclusions}

Thus, as the results of correlation analysis show, speech experience is closely interconnected with personality's individual characteristics. Elements of the internal structure of the speech experience show mutual relationship with emotional and volitional characteristics. Elements of external structures, as it was expected due to the release of their overall function, cause changes in those personal characteristics that determine the interaction of the personality with society. The originality of combining relationships speech experience structural elements with personal characteristics causes, thus, individuality of personality's speech mastering of the world. In personal aspect of speech experience functioning, quantitative level of speech experience organization are replaced with indicators of individual style of combination of personal traits with structural and functional peculiarities of speech experience. So, these individual peculiarities of speech experience lead to individual charac- 
teristics of personality's social connection. It was proved that the features of the social intelligence were largely determined by personal level of speech ability, speech capabilities, speech competence, speech activity and by the level of its organization into system. The analysis of the correlation relations between the levels of organization of the elements of the internal structure of the speech experience and social intelligence capabilities demonstrates the existence of statistically significant. This allows us to assert the mutual influence of speech experience and social intelligence.

The internalization of social contexts is carried out with the help of verbal means, which determines the leading role of speech experience in the development of social intelligence in general. That directs further research into the search for patterns and general peculiarities of interaction between speech experience and social intelligence.

\section{References:}

1. Ivachkevych, E. Z. (2017). Sotsialnyj intellect pedagoga [Social Intelligence of Pedagog] / E. Z. Ivachkevych. - Kyjv : Print-House (in Ukrainian).

2. Mychajlova, E. S. (2006). Metodyka doslidgennja sotsialnogo intellectu [Method of Recearch of the Social Intelligence] / E. S. Mychajlova. - SPb. : "Imaton" (in Russian).

3. Orap, M. O. (2014). Psychologia movlennevogo dosvidu osobustosti [Psychology of the Personality's Speech Experience] / M. O. Orap. - Ternopil : Pidruchnyky and Posibnuky (in Ukrainian).

4. Zasekina, L. V. (2005). Structurno-FUNKTSIONALNA organizatsija intelektu [Structure - Functional Organization of Intelligence] / L. V. Zasekina. Ostrog : Publishing "Ostrog Academy" (in Ukrainian).

5. Argyle, M. (1972) The psychology of interpersonal behavior (2nd ed.). London: Penguin Books.

6. Crowne K.A. (2009) The relationships among social intelligence, emotional intelligence and cultural intelligence. Organization Management Journal, vol. 6, issue 3, pp. 84-89.

7. Gardner, H. (1983) Frames of Mind: The Theory of Multiple Intelligences. New York: Basic Books.

8. Goleman D. (2006) Social Intelligence: The New Science of Human Relationships. New York: Bantam Books.

9. Guilford J.P. (1968) The nature of human intelligence. New York: McGraw-Hill.

10. Ng S.H., Bradac J.J. (1993) Power in language: verbal communication and social influence (Language and Language Behavior) (1st ed.). New York: SAGE Publications.

11. Riggio, R.E. (1986). Assessment of basic social skills. Journal of Personality and Social Psychology, vol.51, pp. 649-660.

12. Sternberg, R.J., et al. (2000). Practical intelligence in everyday life. Cambridge: Cambridge University Press. 